\title{
The role of I-FABP as a biomarker of intestinal barrier dysfunction driven by gut microbiota changes in obesity
}

\author{
Eva Lau ${ }^{1,2,3 \dagger}$, Cláudia Marques ${ }^{3,4 \dagger}$, Diogo Pestana ${ }^{3,4}$, Mariana Santoalha ${ }^{3}$, Davide Carvalho ${ }^{1,2,5}$, Paula Freitas $s^{1,2,5}$ \\ and Conceição Calhau $u^{3,4,6^{*}}$
}

\begin{abstract}
Background: Intestinal fatty-acid binding protein (I-FABP) is expressed in epithelial cells of the mucosal layer of the small intestine tissue. When intestinal mucosal damage occurs, I-FABP is released into the circulation and its plasma concentration increases. In the context of obesity, the gut barrier integrity can be disrupted by dietary fat while intestinal permeability increases.

Objective: To investigate whether intestinal fatty acid binding protein (I-FABP) is a suitable plasma marker of intestinal injury and inflammation in obesity.

Methods: Twelve male Wistar rats were randomly divided into two groups of six animals each: standard (St) and high-fat (HF) diet fed groups for 12 weeks.

Results: HF fed animals developed obesity, insulin resistance and seemed to present increased plasma levels of proinflammatory cytokines (MCP-1 and IL1 $\beta$ ). The gut microbiota composition of these animals was also altered, with lower number of copies of Bacteroidetes, Prevotella spp. and Lactobacillus spp., in comparison with those from St diet group. Fecal lipopolysaccharide (LPS) concentrations tended to be increased in HF fed animals. Intestinal expression of TLR4 seemed to be also increased in HF fed animals suggesting that HF diet-induced dysbiosis may be behind the systemic inflammation observed. However, in contrast to other intestinal inflammatory diseases, plasma I-FABP levels were decreased in HF fed rats whereas I-FABP expression in jejunum tended to be increased.
\end{abstract}

Conclusions: HF diet-induced obesity is characterized by dysbiosis, insulin resistance and systemic inflammation. In this context, plasmatic I-FABP should not be used as a marker of the intestinal barrier dysfunction and the lowgrade chronic inflammatory status.

Keywords: Inflammation, Intestinal fatty acid binding protein, Intestinal permeability, Obesity, Gut microbiota

\section{Background}

The new concepts on the pathophysiology of obesity and insulin resistance highlight the role of intestinal microbiota and intestinal barrier in the development of these disorders [1,2]. Microbiota seems to mediate obesity and associated metabolic disturbances through several

\footnotetext{
* Correspondence: ccalhau@med.up.pt

${ }^{\dagger}$ Equal contributors

${ }^{3}$ Departamento de Bioquímica, Faculdade de Medicina, Universidade do Porto, Porto, Portugal

${ }^{4}$ CINTESIS, Centro de Investigação em Tecnologias e Serviços de Saúde, Porto, Portugal

Full list of author information is available at the end of the article
}

mechanisms including energy storage and metabolic inflammation $[1,3]$. On the other hand, intestinal mucosa plays not only an important part in the absorption of vital nutrients, but also in anatomical/barrier and immune functions, preventing bacterial translocation. High-fat diet changes gut microbiota composition and increases intestinal permeability, by a mechanism associated with a reduced expression of epithelial tight junction proteins [4]. The altered intestinal barrier and the subsequent translocation of bacteria or bacterial products, namely lipopolysaccharide (LPS) are now recognized as key 
mediators of the low-grade inflammation state, which characterize metabolic disorders.

Intestinal fatty-acid binding protein (I-FABP) is an intracellular protein specifically and abundantly expressed in the epithelial cells of the mucosal layer of the small and large intestine tissue [5]. The location of I-FABP in the mature epithelium of villi facilitates its leakage into the circulation from enterocytes when intestinal mucosal damage occurs [5]. It has been shown that I-FABP is released into the circulation following small intestinal mucosal injury and its plasma concentration has been associated with small intestinal diseases - necrotizing enterocolitis and celiac disease [6-8]. Therefore, I-FABP has emerged as a possible non-invasive marker for evaluating gut wall integrity loss and inflammation. Defining new and early non-invasive markers of gut barrier dysfunction might be of great interest in order to manage a safe modulation of the intestinal microbiota before emergence of obesity and associated metabolic diseases.

The aim of this study was to investigate the role of IFABP as a possible plasma marker of intestinal injury and inflammation and its relationship with microbiota dysbiosis in a high-fat diet-induced obesity Rat model.

\section{Methods}

\section{Animals and housing}

Twelve male Wistar rats were purchased from Charles River (Barcelona, Spain) and kept under controlled environmental conditions $\left(22-24{ }^{\circ} \mathrm{C}\right.$ and $12 \mathrm{~h}$ light/dark cycles), for at least 1 week before starting the experiments. Animals, 8 weeks of age, were randomly divided into two groups of six animals each: standard (St) and high-fat (HF) diet group. The diets were respectively "St" (Teklad 2014, Harlan Laboratories, Santiga, Spain) and "HF" with $45 \%$ of energy from lipids and $17 \%$ of energy from sucrose (D12451 Research Diets, New Brunswick, NJ, USA). Animals were subjected to different experimental conditions for a total of 12 weeks. The water and chow were supplied ad libitum. Food and beverage consumption and body weight were monitored weekly, to carefully characterize energy ingestion and weight gain.

At the end of the 12 weeks, food was removed $4-6 \mathrm{~h}$ before sacrifice and the animals were anesthetized with a mixture of ketamine $(50 \mathrm{mg} / \mathrm{kg})$ and medetomidine $(1 \mathrm{mg} / \mathrm{kg})$ and maintained with isoflurane. Meanwhile, using a Quantum/S bioelectrical impedance analyzer (RJL Systems, Akern SRL, Florence, Italy), the body composition of each rat was determined by bioelectrical impedance, according to the procedure already described in the literature [9]. Before perfusion of the vascular compartment with a saline solution $(\mathrm{NaCl} 0.9 \%$, w/v), blood was drawn from the left ventricle into tubes with or without heparin to obtain plasma and serum, respectively. Aliquots were frozen at $-80{ }^{\circ} \mathrm{C}$ until further analysis. Colon and jejunum were dissected, pat dried and snap-frozen in liquid nitrogen. Fresh fecal samples were collected directly from the colon of all animals and snap-frozen in liquid nitrogen. Both tissues and fecal samples were stored at $-80{ }^{\circ} \mathrm{C}$ until further analysis.

Animal handling and housing protocols followed European Union guidelines (Directive 2010/63/EU) for the use of experimental animals in scientific research. The protocol was approved by the Committee on the Ethics of Animal Experiments of the Faculty of Medicine of University of Porto.

\section{Oral glucose tolerance test (OGTT)}

After 7 weeks of treatment, rats were fasted over $5 \mathrm{~h}$ and a baseline blood draw from the saphenous vein was collected for plasma fasting glucose and insulin measurements. Animals were gavaged with a glucose solution of $2 \mathrm{~g} / \mathrm{kg}$ body weight and blood droplets from the saphenous vein were collected to measure glycaemia thereafter at 30, 60, 90 and $120 \mathrm{~min}$. Glucose levels were measured with Precision Xtra Plus test strips and an Optium Xceed device (Abbott Diabetes Care, Ltd., Maidenhead, UK). Plasma insulin levels were measured using a Rat/Mouse Insulin ELISA kit (Merck Milipore, Madrid, Spain). The homeostasis model assessment (HOMA) was used to calculate approximate insulin resistance using the formula: glucose $(\mathrm{mg} / \mathrm{dL}) \times$ insulin $(\mathrm{ng} / \mathrm{mL}) / 405$.

\section{Blood and biochemical analysis}

Biochemical evaluation of plasma was performed at the end of the study at São João Hospital Center Clinical Pathology Department. Routine biochemical parameters were measured using conventional methods with an Olympus AU5400 automated clinical chemistry analyzer (Beckman-Coulter, Izasa, Porto, Portugal).

Plasma content in leptin, adiponectin, monocyte chemoattractant protein-1 (MCP-1), I-FABP and glucagon-like peptide-2 (GLP-2) were determined using, respectively, Rat Leptin ELISA Kit (Merck, Milipore, Madrid, Spain), Human/Mouse/Rat Adiponectin Enzyme Immunoassay Kit (RayBiotech, Norcross, GA, USA), Rat MCP-1 ELISA Kit (RayBiotech, Norcross, GA, USA), Rat (FABP2) ELISA Kit (Shanghai Sunred Biological Technology Co., Ltd, Shangai) and GLP-2 ELISA Kit (Merck, Milipore, Madrid, Spain). Serum interleukin-1 beta (IL-1 $\beta$ ) was determined by Luminex assay using custom Miliplex Rat Kits (Merck Milipore, Madrid, Spain), according to the manufacturer's protocols using the Luminex Xmap Multiplexing Technology platform.

\section{Tissue RNA isolation and qRT-PCR}

Total RNA from jejunum and colon was isolated using NZYol reagent (NZYTech, Portugal) according to 
manufacturer's instructions. RNA samples were treated with DNase I (RQ1 RNase-free DNase; Promega, Portugal). cDNA was synthesized from $1 \mu \mathrm{g}$ of treated mRNA with NZY First-Strand cDNA Synthesis Kit (NZYTech, Portugal). Quantitative real-time polymerase chain reaction (qRT-PCR) was run on Lightcycler96 (Roche Applied Science, Indianapolis, ID, USA). Cycling conditions were as follows: denaturation $\left(95{ }^{\circ} \mathrm{C}\right.$ for $10 \mathrm{~min})$, amplification and quantification $\left(95^{\circ} \mathrm{C}\right.$ for $10 \mathrm{~s}$, annealing temperature for $10 \mathrm{~s}$ and $72{ }^{\circ} \mathrm{C}$ for $10 \mathrm{~s}$, with a single fluorescence measurement at the end of the $72^{\circ}$ $\mathrm{C}$ for a 10-s segment) repeated for 45 cycles and a final melting step with a temperature ramp from 60 to $97{ }^{\circ} \mathrm{C}$. Rat-specific primer sequences (Sigma-Aldrich, St. Louis, MO, USA) used are described in Table 1 . The Cq values obtained were transformed into relative quantification data using the formula $2^{-(\Delta \mathrm{Cq})}$.

\section{DNA extraction from stool}

Genomic DNA was extracted and purified from stool samples using NZY Tissue gDNA Isolation Kit (NZYtech, Lisbon, Portugal) with some modifications. Briefly, feces (170-200 mg) were homogenized in TE buffer (10 mM Tris/HCl; $1 \mathrm{mM}$ EDTA, $\mathrm{pH}$ 8.0) and centrifuged at $4000 \times g$ for $15 \mathrm{~min}$. The supernatant was discarded and the pellet was resuspended in $350 \mu \mathrm{L}$ of buffer NT1. After an incubation step at $95{ }^{\circ} \mathrm{C}$ for $10 \mathrm{~min}$, samples were centrifuged at $11000 \times \mathrm{x}$ for $1 \mathrm{~min}$. Then, $25 \mu \mathrm{L}$ of proteinase $\mathrm{K}$ were added to $200 \mu \mathrm{L}$ of the supernatant for incubation at $70{ }^{\circ} \mathrm{C}$ for $10 \mathrm{~min}$. The remaining steps followed manufacturer's instructions. DNA purity and quantification were assessed with a NanoDrop spectrophotometer (Thermo Scientific, Wilmington, DE, USA).

\section{Microbial analysis of Rat stool by qRT-PCR}

qRT-PCR was performed in sealed 96-well microplates using a LightCycler FastStart DNA Master SYBR Green kit and a LightCycler96 instrument (Roche Applied Science, Indianapolis, ID, USA). Primer sequences (SigmaAldrich, St. Louis, MO, USA) used to target the $16 \mathrm{~S}$

Table 1 Primer sequences and real-time PCR conditions used for gene expression analysis by qRT-PCR

\begin{tabular}{lll}
\hline Gene name & Primer Sequence $\left(5^{\prime}\right.$-3') & AT \\
\hline I-FABP & ATGGAAAGGAGCTGATTGCT & $59^{\circ} \mathrm{C}$ \\
& TTGGCCTCCACTCCTTCATA & \\
TLR4 & GATGCCTCTCTTGCATCTGG & $60^{\circ} \mathrm{C}$ \\
& TCATGAGGGATTTGCTGAGA & \\
GAPDH & GGCATCGTGGAAGGGCTCATGAC & $62^{\circ} \mathrm{C}$ \\
& ATGCCAGTGAGCTTCCCGTTCAGC & \\
\hline
\end{tabular}

$A T$ annealing temperature, GAPDH glyceraldehyde-3-phosphate dehydrogenase (housekeeping gene), I-FABP intestinal fatty acid binding protein, TLR4 Toll-like receptor 4
rRNA gene of the bacteria and the conditions for PCR amplification reactions were previously described in Marques $C$ et al. [10]. To verify the specificity of the amplicon, a melting curve analysis was performed via monitoring SYBR Green fluorescence in the temperature ramp from 60 to $97{ }^{\circ} \mathrm{C}$. Data were processed and analyzed using the LightCycler software (Roche Applied Science, Indianapolis, ID, USA). Standard curves were constructed using serial tenfold dilutions of bacterial genomic DNA, according to the following webpage http://cels.uri.edu/gsc/cndna.html. Bacterial genomic DNA used as a standard was obtained from DSMZ (Braunschweig, Germany). Genome size and the copy number of the 16S rRNA gene for each bacterial strain used as a standard was obtained from NCBI Genome database (www.ncbi.nlm.nih.gov). Data are presented as the mean values of duplicate PCR analysis.

\section{Fecal LPS quantification}

Quantification of LPS was performed using the ChromoLimulus Amebocyte Lysate (Chromo-LAL) reagent (Associates of Cape Cod, Inc., Falmouth, MA, USA). Briefly, $1 \mathrm{~mL}$ of sterile saline solution $(\mathrm{NaCl} 0.9 \%)$ was added to $100 \mathrm{mg}$ feces, vortexed and centrifuged $(10 \mathrm{~min}, 10000 \mathrm{~g}$, $4{ }^{\circ} \mathrm{C}$ ) twice. Total supernatant (fecal water) was filtered with $0.45 \mu \mathrm{m}$ filter and then with $0.22 \mu \mathrm{m}$ filter. Fecal water and Chromo-LAL (1:1) were incubated at $37^{\circ} \mathrm{C}$ for $20 \mathrm{~min}$ and absorbance was read every $10 \mathrm{~s}$ at $405 \mathrm{~nm}$.

\section{Statistical analysis}

Values are expressed as the arithmetic mean \pm standard error of the mean (SEM). Given the small sample size, a non-parametric test (Mann-Whitney test) was used to analyze the differences between St and HF groups. Correlation between variables was established using twotailed Pearson's correlation test. The differences were considered statistically significant when $P<0.05$. All statistical analyses were performed using GraphPad Prism 6 statistical software (GraphPad Software Inc., La Jolla, CA, USA).

\section{Results}

\section{Energy and metabolic parameters}

Energy intake was increased in the HF diet fed group when compared with St diet fed group $(72.60 \pm 0.15$ vs. $57.9 \pm 0.47 \mathrm{kcal} /$ day, $P<0.05)$. Consequently, HF diet fed animals gained more weight during the 12 weeks of the study (205.5 \pm 37.8 vs. $134.7 \pm 22.5 \mathrm{~g}, P<0.05)$ (Table 2$)$. To assess whether these differences in weight gain were related to alterations in adiposity, we decided to evaluate the body composition of the animals of both groups. Our results showed that rats under HF diet had more body fat mass comparing to rats under St diet (206.3 \pm 21.03 vs. $166.8 \pm 21.26, P<0.05)$. 
Table 2 Energy ingestion, body composition and metabolic parameters of Wistar rats fed either with standard (St) or high-fat (HF) diet during 12 weeks

\begin{tabular}{lcll}
\hline & St & HF & $P$ value \\
\hline Energy ingested (Kcal/day) & $57.9 \pm 0.5$ & $72.60 \pm 0.1$ & $<0.05$ \\
Weight gain (g) & $134.7 \pm 9.2$ & $205.5 \pm 15.4$ & $<0.05$ \\
Fat mass (g) & $166.8 \pm 8.7$ & $206.3 \pm 8.6$ & $<0.05$ \\
Total cholesterol (mg/dL) & $64.3 \pm 3.1$ & $69.8 \pm 6.6$ & 0.78 \\
Triglycerides (mg/dL) & $79.3 \pm 11.5$ & $79.2 \pm 8.3$ & 0.83 \\
Leptin (ng/mL) & $6.2 \pm 1.2$ & $19.1 \pm 2.6$ & $<0.05$ \\
GLP-2 & $7.0 \pm 0.4$ & $9.8 \pm 0.8$ & $<0.05$ \\
\hline
\end{tabular}

Values are presented as mean \pm SEM ( $n=6$ rats per group). GLP-2 glucagon-like peptide-2

To assess glycemic response, we performed an oral glucose tolerance test (OGTT). The total area under the curve (AUC) of the glycemic response was increased by HF diet feeding (Fig. 1a, b). In addition, HF diet fed rats had almost two fold less insulin sensitivity, as determined by homeostasis model assessment (HOMA) of insulin resistance (Fig. 1c).

We also examined plasma concentrations of leptin, which has postulated roles in obesity and insulin action (Table 2). HF diet fed group presented significantly higher leptin levels $(19.1 \pm 2.6$ vs. $6.2 \pm 1.2, P<0.05)$ than St diet fed group.

Total cholesterol and triglycerides did not differ between groups (Table 2).

\section{Gut microbiota, LPS and inflammatory status}

To study whether HF diet could induce modifications within the intestinal microbiota, we quantified some of the main bacterial groups presented in fecal samples. Analysis of the bacterial $16 \mathrm{~S}$ rDNA revealed that, at the phylum level, animals from the HF diet group were characterized for having lower Bacteroidetes and higher Firmicutes to Bacteroidetes ratio (Table 3). Firmicutes/ Bacteroidetes ratio was positively correlated with weight gain $(r=0.829, P<0.05)$ and AUC $(r=-0.723, P<0.05)$ while Bacteroidetes were negatively correlated with weight gain $(r=-0.800, P<0.05)$ and AUC $(r=-0.716 P<0.05)$.

HF diet feeding also resulted in a decrease in the number of copies of Prevotella spp. and Lactobacillus spp. (Table 3). We further determined whether fecal LPS levels could be altered as a result of the changes in the gut microbiota. Our data showed that fecal LPS levels seemed to be more elevated in HF diet fed animals (Fig. 2a). In addition, LPS levels were positively correlated with Firmicutes/Bacteroidetes ratio $(r=0.787, P<0.05)$ and negatively correlated with Bacteroidetes $(r=-0.670, P<0.05)$.

Afterwards, we evaluated the colonic expression of tolllike receptor 4 (TLR4) which is capable to recognize LPS. In agreement with our LPS findings, TLR4 expression

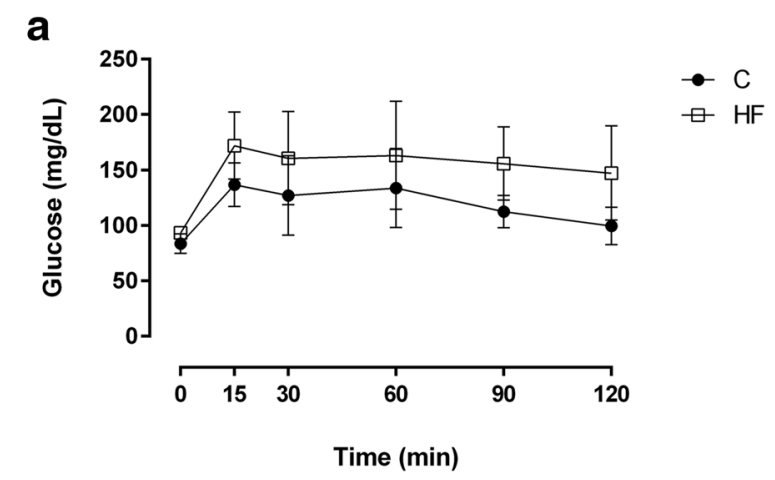

b

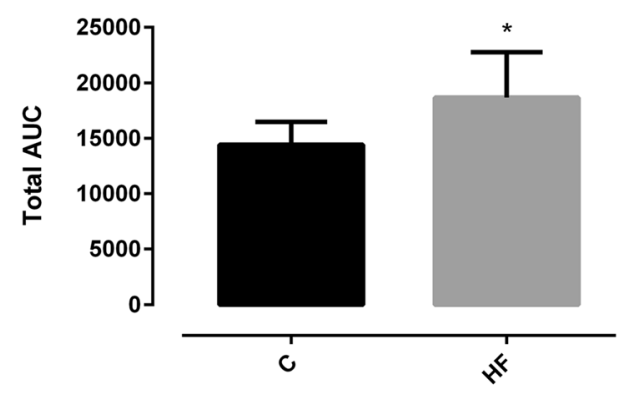

C

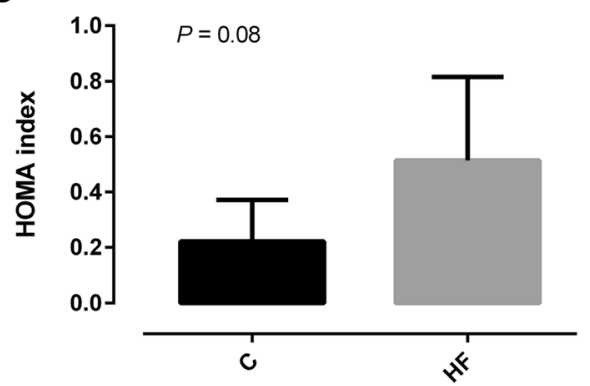

Fig. 1 Glycaemic response during oral glucose tolerance test (a), total area under the curve (AUC) (b) and homeostasis model assessment (HOMA) (c) of Wistar rats after 7 weeks of feeding either with standard (St) or high-fat (HF) diet. HOMA was calculated using the formula: fasting glucose $(\mathrm{mg} / \mathrm{dL}) \times$ fasting insulin $(\mathrm{ng} / \mathrm{mL}) / 405$. Data are presented as mean $\pm \operatorname{SEM}$ ( $n=6$ rats per group). ${ }^{*} P<0.05$ vs respective St diet group

tended to be increased in the colon of animals fed with HF diet (Fig. 2b). Next, we investigated whether HF diet and microbiota changes were associated with systemic inflammation. In accordance, the chemokines MCP-1 and Il-1 $\beta$ appeared to be more elevated in the plasma of HF diet fed rats (Fig. 2c, d). LPS was positively correlated with MCP-1 $(r=0.726, P<0.05)$.

\section{I-FABP and GLP-2 in high fat-diet induced obesity}

To understand the relationship between I-FABP, HF diet feeding and systemic inflammation we quantified plasma I-FABP levels and its intestinal expression in both groups of rats. Surprisingly, plasma I-FABP levels were decreased after HF diet feeding (Fig. 3a). On the other 
Table 3 Quantification of gut microbiota phyla, genera and species in different experimental groups

\begin{tabular}{llll}
\hline & St & HF & $P$ value \\
\hline Firmicutes/Bacteroidetes & $1.03 \pm 0.01$ & $1.20 \pm 0.03$ & $<0.05$ \\
Firmicutes & $6.61 \pm 0.08$ & $6.50 \pm 0.09$ & 0.35 \\
Bacteroidetes & $6.43 \pm 0.10$ & $5.45 \pm 0.15$ & $<0.05$ \\
Bacteroides spp. & $4.36 \pm 0.23$ & $4.15 \pm 0.50$ & 0.18 \\
Prevotella spp. & $3.59 \pm 0.29$ & $2.21 \pm 0.13$ & $<0.05$ \\
Lactobacillus spp. & $4.86 \pm 0.27$ & $3.77 \pm 0.17$ & $<0.05$ \\
Clostridium leptum & $5.53 \pm 0.04$ & $5.43 \pm 0.12$ & 0.65 \\
Bifidobacterium spp. & $2.00 \pm 0.24$ & $2.12 \pm 0.22$ & 0.75 \\
\hline
\end{tabular}

Values are presented as mean \pm SEM and expressed as $\log _{10} 16 \mathrm{~S}$ rRNA gene copies/ $20 \mathrm{ng}$ of DNA ( $n=6$ rats per group). HF high-fat diet group, St standard diet group

hand, I-FABP relative expression in jejunum tended to be higher in HF diet fed rats (Fig. 3b) which could be considered an adaptive response to the increased dietary fat content of the diet. To determine intestinotrophic status of the animals we quantified GLP-2 plasma levels. GLP-2 plasma levels were significantly increased after HF diet feeding (Table 2). GLP-2 and intestinal expression of I-FABP seemed positively correlated with energy ingested $(r=0.719, P<0.05$ and $r=0.770, P=0.07$, respectively).

To determine whether I-FABP plasma levels could be used as a marker of the metabolic alterations and inflammatory status associated with obesity, we evaluated the correlation between plasma I-FABP and host metabolic and inflammatory parameters (Fig. 4). Plasma I-
FABP levels were negatively correlated with fecal LPS $(r=-0.806, P<0.05)$ and IL-1B $(r=-0.623, P<0.05)$.

\section{Discussion}

Several studies have provided compelling evidence suggesting an association between gut microbiome dysbiosis, obesity and low-grade inflammatory state [11].

In consonance with previous reports, we found that the animals fed with HF diet had lower Bacteroidetes and higher Firmicutes to Bacteroidetes ratio [10]. This dysbiosis pattern might lead to an increased capacity of harvesting energy from food [12, 13]. HF diet also induced obesity and may have triggered intestinal inflammation since fecal levels of LPS and TLR4 expression tended to be increased in the animals fed with this diet. TLR4 is the LPS primary receptor that mediates its proinflammatory effects [14]. As a result, HF fed animals seemed to present higher plasma levels of proinflammatory cytokines and developed insulin resistance. As supported by other authors, it appears to be a causative role for the gut bacteriainduced proinflammatory state to the development of weight gain and insulin resistance in rats under HF diet $[15,16]$. In this context, the gut barrier has an important role in the prevention of LPS leakage from the intestinal lumen to the portal blood. However, in animal models of diet-induced obesity, intestinal barrier function seems to be compromised [17].

In different intestinal diseases, I-FABP has emerged as a potential biomarker of intestinal barrier dysfunction
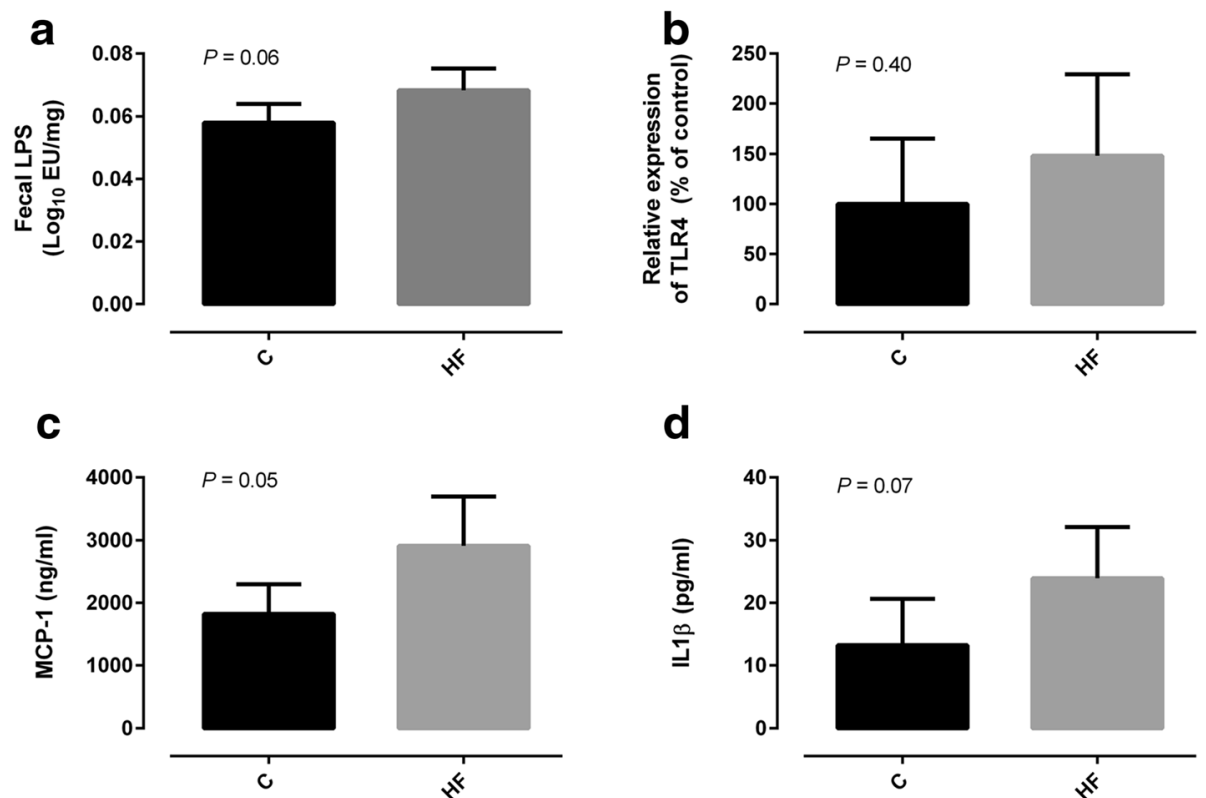

Fig. 2 Fecal lipopolysaccharide (LPS) (a), intestinal expression of toll-like receptor 4 (TLR4) (b), plasma monocyte chemoattractant protein-1 $(\mathrm{MCP}-1)(\mathbf{c})$ and serum interleukin-1 beta $(\mathrm{IL}-1 \beta)(\mathbf{d})$ of Wistar rats fed either with standard (St) or high-fat (HF) diet during 12 weeks. Data are presented as mean \pm SEM ( $n=3-6$ rats per group). ${ }^{*} P<0.05$ vs respective St diet group 

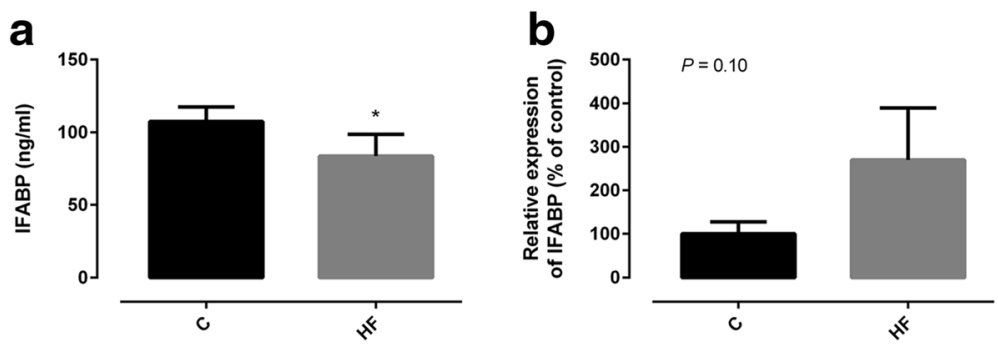

Fig. 3 Levels of plasma (a) and intestinal expression of intestinal fatty-acid binding protein (I-FABP) (b) of Wistar rats fed either with standard (St) or high-fat (HF) diet during 12 weeks. Data are presented as mean \pm SEM $\left(n=3-6\right.$ rats per group). ${ }^{*} P<0.05$ vs respective St diet group

$[18,19]$. Basal I-FABP plasma levels may reflect the physiological turnover rate of enterocytes, whereas elevated levels might indicate intestinal epithelial cell damage [20]. Nevertheless, in the case of obesity-associated metabolic diseases, the existent data about I-FABP and intestinal barrier dysfunction is limited.

Verdam et al. have reported that chronically elevated glucose levels in obese individuals were associated with increased enterocyte loss, assumed by the increase on IFABP levels [21]. It was therefore speculated that the increased enterocyte loss observed in subjects with chronic hyperglycemia might had contributed to the impaired intestinal barrier function, thereby promoting endotoxin-induced low-grade inflammation. However, increased I-FABP levels could also be a result of an increased production of I-FABP by enterocytes rather than enterocyte loss.

Interestingly, in our study we found that the relative expression of I-FABP tended to be increased in HF diet fed rats. As intestinal absorption capacity can be adapted to the dietary fat content, we hypothesize that HF diet may had up-regulated several genes known to play an important role in long-chain fatty acids uptake such as I-FABP [22]. The animals fed with HF diet also showed increased GLP-2 levels. GLP-2 is a 33 amino acid peptide associated with intestinal growth and adaptation in a variety of pathological conditions [23]. As suggested by other authors, GLP2/GLP2R system may be increased after HF diet to further promote fat absorption in the intestine [23]. On the other hand, the inflammatory state induced by microbiota changes after HF diet feeding might have increased GLP-2 production in order to improve the mucosal barrier integrity and, therefore, blunt the inflammatory stress [2].

\section{Conclusions}

To our best knowledge, this is the first study demonstrating that, inversely to what happens in other

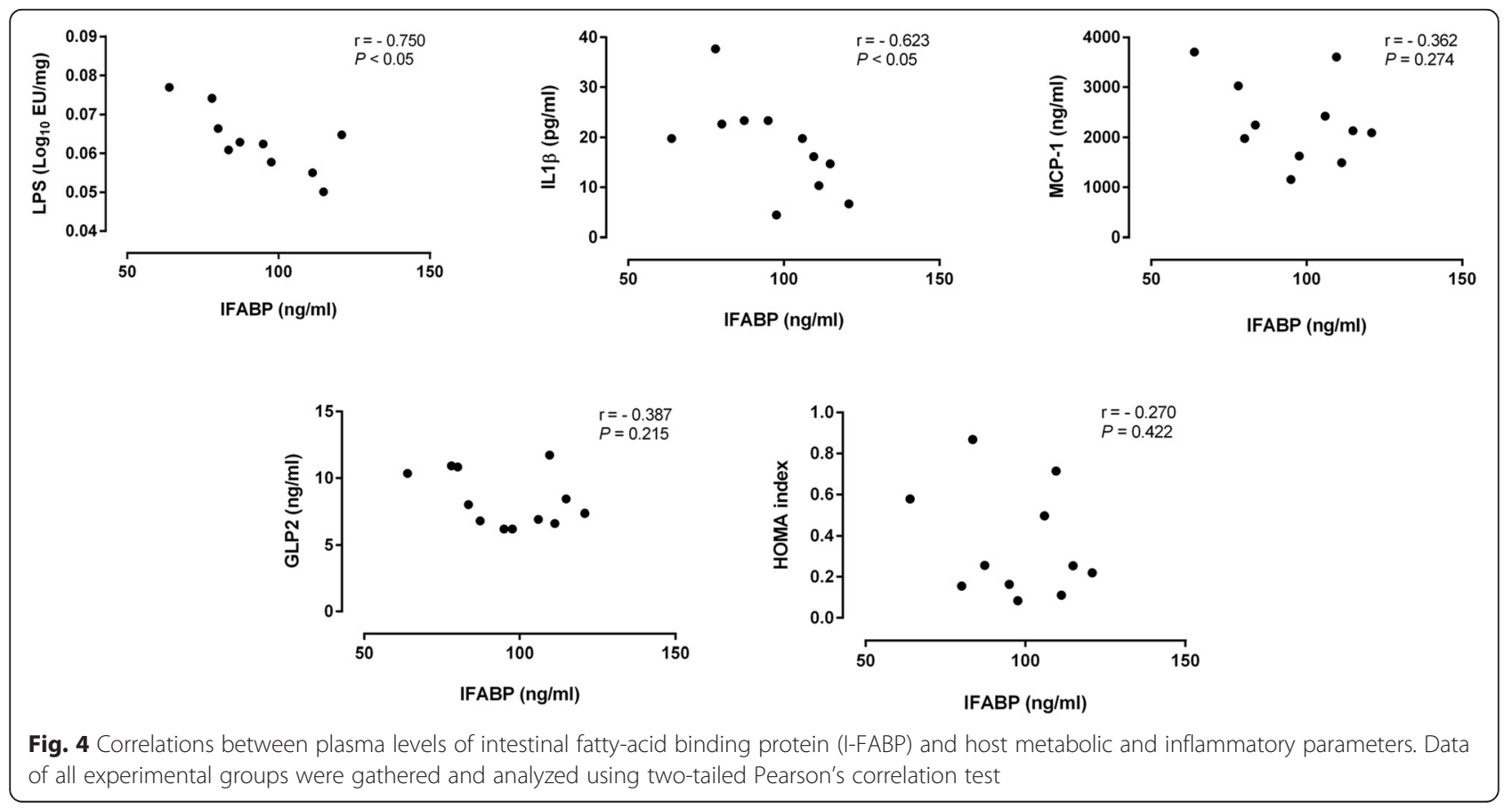


intestinal inflammatory diseases, plasma I-FABP does not positively correlates with the inflammatory status presented in obesity. Instead, I-FABP is decreased in plasma but probably increased in jejunum in order to face dietary fat content. The search for noble biomarkers has to continue since it is extremely important to anticipate the progression of obesity-associated metabolic diseases and, thus, allowing the prevention or monitoring of the therapeutic strategies use.

\section{Abbreviations \\ AUC: area under the curve; GLP-2: glucagon-like peptide-2; HF: high-fat; HOMA: homeostasis model assessment; I-FABP: intestinal fatty-acid binding protein; IL-1ß: interleukin-1 beta; LPS: lipopolysaccharide; MCP-1: monocyte chemoattractant protein-1; OGTT: oral glucose tolerance test; qRT-PCR: quantitative real-time polymerase chain reaction; St: standard; TLR4: toll-like receptor 4.}

\section{Competing interests}

The authors declare that they have no competing interests.

\section{Authors' contributions}

EL, CM, DC, PF and CC designed the study. EL, CM, DP and MS performed laboratory work. EL and CM analyzed and interpreted data and wrote the manuscript; DC, PF, CC critically revised the manuscript. All authors have read and approved the final manuscript.

\section{Funding}

Financial support from Fundação para Ciência e Tecnologia (PTDC/AGR-TEC/ 2227/2012 and SFRH/BD/93073/2013) is gratefully acknowledged.

\section{Author details}

${ }^{1}$ Serviço de Endocrinologia, Diabetes e Metabolismo, Centro Hospitalar São João, Porto, Portugal. ${ }^{2}$ Instituto de Investigação e Inovação em Saúde, Porto, Portugal. ${ }^{3}$ Departamento de Bioquímica, Faculdade de Medicina,

Universidade do Porto, Porto, Portugal. ${ }^{4}$ CINTESIS, Centro de Investigação em Tecnologias e Serviços de Saúde, Porto, Portugal. ${ }^{5}$ Departamento de Medicina, Faculdade de Medicina, Universidade do Porto, Porto, Portugal. ${ }^{6}$ Nutrição e Metabolismo, NOVA Medical School|Faculdade de Ciências Médicas, Universidade NOVA de Lisboa, Lisboa, Portugal.

Received: 8 February 2016 Accepted: 19 April 2016

Published online: 30 April 2016

\section{References}

1. Cani PD, Amar J, Iglesias MA, Poggi M, Knauf C, Bastelica D, Neyrinck AM, Fava F, Tuohy KM, Chabo C, Waget A, Delmée E, Cousin B, Sulpice T, Chamontin B, Ferrières J, Tanti JF, Gibson GR, Casteilla L, Delzenne NM, Alessi MC, Burcelin R. Metabolic endotoxemia initiates obesity and insulin resistance. Diabetes. 2007:56:1761-72.

2. Cani PD, Possemiers S, Van de Wiele T, Guiot Y, Everard A, Rottier O, Geurts L, Naslain D, Neyrinck A, Lambert DM, Muccioli GG, Delzenne NM. Changes in gut microbiota control inflammation in obese mice through a mechanism involving GLP-2-driven improvement of gut permeability. Gut. 2009;58:1091-103.

3. Bäckhed F, Ding H, Wang T, Hooper LV, Koh GY, Nagy A, Semenkovich CF, Gordon JI. The gut microbiota as an environmental factor that regulates fat storage. Proc Natl Acad Sci U S A. 2004;101:15718-23.

4. Cani PD, Bibiloni R, Knauf C, Waget A, Neyrinck AM, Delzenne NM, Burcelin R. Changes in gut microbiota control metabolic endotoxemiainduced inflammation in high-fat diet-induced obesity and diabetes in mice. Diabetes. 2008:57:1470-81.

5. Pelsers MMA, Namiot Z, Kisielewski W, Namiot A, Januszkiewicz M, Hermens WT, Glatz JF. Intestinal-type and liver-type fatty acid-binding protein in the intestine. Tissue distribution and clinical utility. Clin Biochem. 2003:36:529-35.

6. Adriaanse MPM, Tack GJ, Passos VL, Damoiseaux JGMC, Schreurs MWJ, van Wijck K, Riedl RG, Masclee AAM, Buurman WA, Mulder CJ, Vreugdenhil ACE. Serum I-FABP as marker for enterocyte damage in coeliac disease and its relation to villous atrophy and circulating autoantibodies. Aliment Pharmacol Ther. 2013;37:482-90.

7. Vreugdenhil AC, Wolters VM, Adriaanse MP, Van den Neucker AM, van Bijnen AA, Houwen R, Buurman WA. Additional value of serum I-FABP levels for evaluating celiac disease activity in children. Scand J Gastroenterol. 2011;46:1435-41.

8. Thuijls G, Derikx JPM, van Wijck K, Zimmermann LJ, Degraeuwe PL, Mulder $T L$, Van der Zee DC, Brouwers HAA, Verhoeven BH, van Heurn LWE, Kramer BW, Buurman WA, Heineman E. Non-invasive markers for early diagnosis and determination of the severity of necrotizing enterocolitis. Ann Surg. 2010:251:1174-80.

9. Rutter K, Hennoste L, Ward LC, Cornish BH, Thomas BJ. Bioelectrical impedance analysis for the estimation of body composition in rats. Lab Anim. 1998;32:65-71.

10. Marques C, Meireles M, Norberto S, Leite J, Freitas J, Pestana D, Faria A, Calhau C. High-fat diet-induced obesity Rat model: a comparison between Wistar and Sprague-Dawley Rat. Adipocyte. 2015;3945(July):1-11.

11. Lau E, Carvalho D, Pina-Vaz C, Barbosa JA, Freitas P. Beyond gut microbiota: understanding obesity and type 2 diabetes. Hormones. 2015;14:358-69.

12. Bäckhed F, Manchester JK, Semenkovich CF, Gordon Jl. Mechanisms underlying the resistance to diet-induced obesity in germ-free mice. Proc Natl Acad Sci U S A. 2007:104:979-84

13. Ley RE, Bäckhed F, Turnbaugh P, Lozupone CA, Knight RD, Gordon Jl. Obesity alters gut microbial ecology. Proc Natl Acad Sci U S A. 2005;102:11070-5

14. Chow JC, Young DW, Golenbock DT, Christ WJ, Gusovsky F. Toll-like Receptor-4 Mediates Lipopolysaccharide-induced Signal Transduction. J Biol Chem. 1999;274(16):10689-92.

15. Wright ORL, Netzel GA, Sakzewski AR. A randomized, double-blind, placebo-controlled trial of the effect of dried purple carrot on body mass, lipids, blood pressure, body composition, and inflammatory markers in overweight and obese adults: the QUENCH trial. Can J Physiol Pharmacol. 2013:91:480-8.

16. Kaliannan K, Hamarneh SR, Economopoulos KP, Nasrin Alam S, Moaven O, Patel P, Malo NS, Ray M, Abtahi SM, Muhammad N, Raychowdhury A, Teshager A, Mohamed MMR, Moss AK, Ahmed R, Hakimian S, Narisawa S, Millán JL, Hohmann E, Warren HS, Bhan AK, Malo MS, Hodin RA. Intestinal alkaline phosphatase prevents metabolic syndrome in mice. Proc Natl Acad Sci U S A. 2013;1 10:7003-8.

17. Stenman LK, Holma R, Korpela R. High-fat-induced intestinal permeability dysfunction associated with altered fecal bile acids. World J Gastroenterol. 2012:18:923-9.

18. Schellekens DHSM, Grootjans J, Dello SAWG, van Bijnen AA, van Dam RM, Dejong CHC, Derikx JPM, Buurman WA. Plasma intestinal fatty acid-binding protein levels correlate with morphologic epithelial intestinal damage in a human translational ischemia-reperfusion model. J Clin Gastroenterol. 2014;48:253-60

19. Schurink M, Kooi EMW, Hulzebos CV, Kox RG, Groen H, Heineman E, Bos AF, Hulscher JBF. Intestinal fatty acid-binding protein as a diagnostic marker for complicated and uncomplicated necrotizing enterocolitis: a prospective cohort study. PLoS One. 2015;10, e0121336.

20. Bischoff SC, Barbara G, Buurman W, Ockhuizen T, Schulzke JD, Serino M, Tilg $\mathrm{H}$, Watson A, Wells J. Intestinal permeability i a new target for disease prevention and therapy. BMC Gastroenterol. 2014;14:189.

21. Verdam FJ, Greve JWM, Roosta S, van Eijk H, Bouvy N, Buurman WA, Rensen SS. Small intestinal alterations in severely obese hyperglycemic subjects. J Clin Endocrinol Metab. 2011;96:E379-83.

22. Petit V, Arnould L, Martin P, Monnot MC, Pineau T, Besnard P, Niot I. Chronic high-fat diet affects intestinal fat absorption and postprandial triglyceride levels in the mouse. J Lipid Res. 2007;48:278-87.

23. Baldassano S, Amato A, Cappello F, Rappa F, Mulè F. Glucagon-like peptide-2 and mouse intestinal adaptation to a high-fat diet. J Endocrinol. 2013;217:11-20. 